\title{
Trends in Satellite Communications
}

\author{
Enrico Del Re, Luca Simone Ronga, Laura Pierucci \\ Dipartimento di Elettronica e Telecomunicazioni \\ Università degli Studi di Firenze \\ Via di Santa Marta 3, 50136 Firenze - Italy
}

\begin{abstract}
Satellite communications exhibit a tremendous growth due to three main elements: interactivity, coverage and bandwidth asymmetry. The paper will explore some of the current trends of the development of next generation satellite systems by analyzing several aspects: from orbital tracks to technology, from services to business opportunities. Both positive and negative experiences are considered in order to fully understand the most probable future scenarios in the satellite area.
\end{abstract}

Index Terms — Satellite communications.

\section{INTRODUCTION}

Satellite communications exhibit a tremendous growth due to three main elements: interactivity, coverage and bandwidth asymmetry. The fast changing of the interactions between these elements let us assist to a complete and radical modifications in the development of next generation communication systems.

Satellites provide several services, including broadcasting of information, acquisition and delivery of weather images, positioning, military surveillance, worldwide phone services and earth observing. If we focus on communication satellite systems, the most popular service is the analog video broadcasting. Most of the 871 current operational geostationary satellites around the globe provide video broadcasting services. The digital version of this service, Digital Video Broadcast over Satellite (DVB-S) is rapidly developing on the main world areas. The digitalization of the video broadcasting service had a massive impact on the development of future generation systems. Interactivity has become possible for digital satellite terminals, allowing a large number of users to access bi-directional services. The DVB-S transport is designed to carry packet data traffic along with real time video, providing the users with a large downstream channel suitable for WEB browsing and file downloading. The current DVB-S WEB services rely on terrestrial networks to complete the bi-directional path to the content provider. Improvements in radio technology and the exploitation of Ka Band will soon let the DVB-S systems migrate toward the fully satellite version, DVB-RCS (DVB Return Channel via Satellite) where the users can access a bidirectional satellite channels. More details will be given in the next sections.

Another reason of the increasing interest in the satellite delivery of services is the coverage aspect. Terrestrial networks will shortly provide great amounts of bandwidth to the end user. A satellite system is usually expensive and characterized by large time-to-market period, nevertheless it receives a justification of its deployment for its ability to provide a coverage on a per-area basis, rather than a per-user basis. This is essential to modern market models, where the delivery of services can be subject to fast variations due to competition and market strategies. The investments on a satellite systems are distributed over a large geographical area and the provided services can be easily moved from a set of users to another without any additional costs. The competing terrestrial network provider must rely on the fidelity of its user in order not to waste the heavy last mile investments.

The last element considered essential to the future development of next satellite systems is the bandwidth asymmetry. The large scale evolution of services moves in the direction of a marked separation of the uplink and downlink traffic. Full-duplex voice and low speed interactive services, e.g. Telnet, are characterized by a symmetric bandwidth demand. Video on demand, WEB, file transfers, distributed games, database accesses require different constraints in bandwidth between the up-link and the downlink. The most efficient way to deal with time varying asymmetric bandwidth demand is the use of a common resource dynamically shared among users. This is again a work for satellites. The radio medium is massively distributed and dynamic resource allocation techniques can easily take advantage from the statistic characteristics of the traffic demands.

The paper will explore some of the current trends of the development of next generation satellite systems. The analysis will deal with different aspects, such as satellite positioning and orbital tracks in section II, technology aspects in section III, future services in section IV and new market perspectives in section $\mathrm{V}$.

\section{TRENDS IN CONSTELLATIONS}

The satellite orbital position has a great impact on the major parameters of a satellite system. Cost, type and quality of service, coverage area and technology of terminals are affected by the orbital characteristics of the satellite. The most popular track is represented by GEO-stationary orbit, placed in the equatorial plane at a height of about $35900 \mathrm{~km}$. The height and inclination make it possible for the satellite to be synchronous with the earth motion and the considerable height provides a very large coverage area. The drawbacks 
related to the satellite-to-earth distance are the need for directive, and so large, antennas on the satellite, the need for high transmission power from the earth terminals and the large propagation delay (earth-satellite-earth jump of about $250 \mathrm{~ms}$ ), that, in conjunction with large bandwidth has a negative effect on the transmission of data when flow control algorithm are employed (e.g. TCP/IP protocol stack).

By reducing the distance between the satellite and the earth the geo synchronicity is lost, and a specific geographical coverage is obtained with more satellites that in turn take over the service until there is visibility between the satellite and the user on earth. This constellation, often referred to as LEO (Low Earth Orbit), encompasses satellite placed at an height ranging from 700 to $1500 \mathrm{~km}$. Due to the reduced propagation time and loss this systems are mainly employed for voice services. Among this systems are Globalstar and Iridium.

Globalstar is founded by Loral Corp. and Qualcomm Inc. to offer low-cost, high-quality global telephony and other digital telecommunications services to areas currently underserved or not served by existing wireline and cellular telecommunications systems. The satellites were launched into 8 orbital planes, with 6 satellites in each plane to provide continuous global coverage.

The Iridium system, a constellation of 66 LEO satellites was founded by Iridium LLC, an international consortium of 17 investor organizations representing telecommunications and industrial companies worldwide.

The Iridium system was founded by Iridium LLC, an international consortium of 17 investor organizations representing telecommunications and industrial companies worldwide. The high costs of operation and the delay in the delivery of the service brought the system to bankruptcy in 1999. After a series of market operations the system is now to be upgraded for classified services, voice and paging and it is now operating under a private ownership.

Iridium and Globalstar story show how difficult is to deal with high costly satellite communication systems. The lesson learned is that when the technology is expensive it should be wiser to find a right balance between quality and management costs. In the case of satellite constellations, LEO at higher altitudes or MEO are to be preferred for voice services since the satellites for a global coverage are less.

In Globalstar or in the proposed ICO and Skybridge each satellites on circular orbit retransmitted the traffic received from the user terminal to a ground gateway while systems utilizing direct intersatellite links (ISLs) among neighboring satellites help to construct a network in sky and offer new challenges [6]. Iridium uses ISLs but also the new proposed systems: Astrolink (9 GEO satellites in Ka band), Spaceway (4/21 GEO satellites in Ka band), and Teledesic (288 LEO satellites in Ka band) starting on 2002.

The utilize of ISL will allow to transport a high amount of TCP traffic providing high speed Internet service but also at
LEO/MEO altitude, will form a complex dynamic network needing complex routing topology.

In the European COST 252 Action "Evolution of satellite personal communication from $2^{\text {nd }}$ to future generation systems" different satellite constellation have been selected [7][8]. Simulation results [8] for inclined MEO, polar MEO, polar LEO orbits with different number of satellites show MEO satellite constellations with satellite numbers ranging from 10 to 30 can provide coverage to users at minimum elevation angles $\varepsilon_{\min }$ (the most limiting parameter) of $40^{\circ}$ [8], so in terms of elevation angle performance it can be seen that MEO are superior to LEO, although at the cost of higher delay and propagation attenuation.

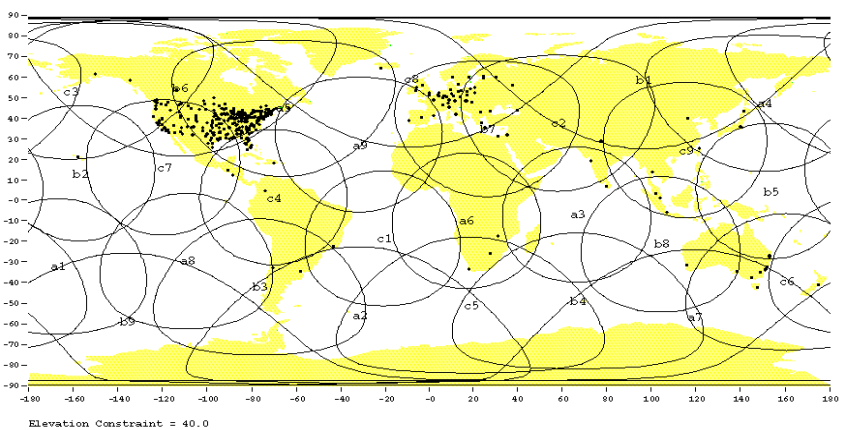

(a) - Inclined MEO

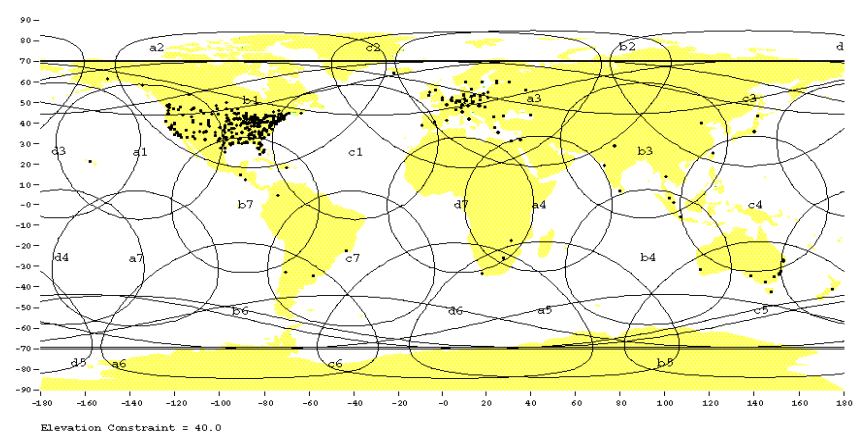

(b) - Polar MEO

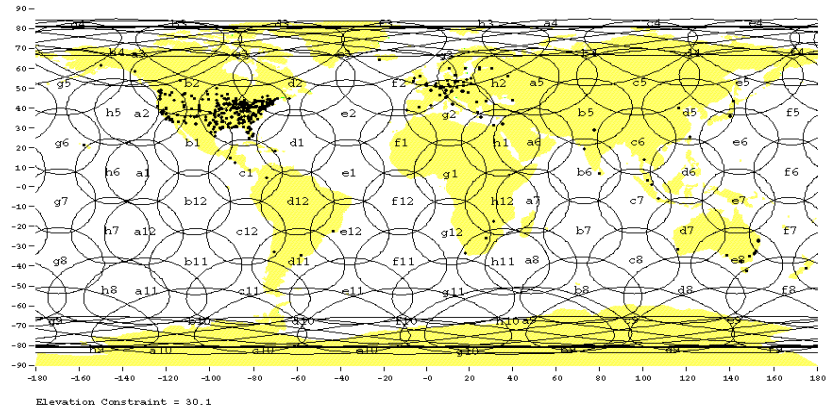

(c) - Polar LEO

Fig. 1 Coverage obtained by the selected satellite constellations 
Other constellations exist. For example High Elliptic Orbits (HEO) satellite characterized by a large difference between the perigee and apogee such as the commercial Ellipso and Pentriad proposal can offer communication service only while the satellites moved slowly around the high apogeee.. The development of such system, however, remain confined to specific and local services and probably this solution will have a limited use for telecommunication purposes.

\section{TRENDS IN TECHNOLOGY}

Exponential grow charts are frequently used to present technology improvements over time. There are important distinction, however, in the grow rates and often the interactions between market and research leads to unattended trends or marked forks between technology potential and its real implementation.

\section{1) Propagation}

The satellite-to-earth propagation path ranges from 700 to $200000 \mathrm{~km}$ with frequencies in the SHF and EHF bands (3$300 \mathrm{GHz}$ ), usually in Line of Sight (LOS). Due to the large distance the frequencies used for dense satellite communication services cannot be used for terrestrial services to avoid interference so the choice for available frequency narrows. Some popular commercial frequency bands for satellite communications are listed in Table III.

Table III

Satellite Commercial Frequency Allocation

\begin{tabular}{l|l}
\hline S-Band & $1700-3000 \mathrm{MHz}$ \\
\hline C-Band & $3700-4200 \mathrm{MHz}$ \\
\hline Ku1-Band & $10.9-11.75 \mathrm{GHz}$ \\
\hline Ku2-Band & $11.75-12.5 \mathrm{GHz}(\mathrm{DBS})$ \\
\hline Ku3-Band & $12.5-12.75 \mathrm{GHz}$ \\
\hline Ka1-Band & $18.0-20.0 \mathrm{GHz}$ \\
\hline Ka2- Band & $29.0-30.0 \mathrm{GHz}$ \\
\hline
\end{tabular}

$\mathrm{Ku}$ Band is mainly used for TV broadcasting services, both analog and digital. The dense utilization and carrier spacing leave no room for broadband interactive services.

For this reasons $\mathrm{Ka}$ band is considered to be the right choice for multimedia bi-directional services. Unfortunately Ka1 band is close to the water vapor absorption window at $22 \mathrm{GHz}$ that impairs satellite transmissions during some meteorological events. Several methods have been studied to fight against weather driven fading in Ka. The larger availability of on-board processing promotes adaptive power allocation strategies. Research in this field is conducted by ASI with the DAVID project [3].

\section{2) Antenna}

The proposal to use $\mathrm{Ka}$ band potentially offers higher bandwidth than $\mathrm{Ku}$ band and very small antennas for the residential user can be used.
LEO satellites permits low power earth terminals and omnidirectional antennas so, for voice services at limited bit rates, handheld device can be implemented, like the Iridium and Globalstar devices .

For higher bit-rates, however, a greater bit energy is required at receiver so, to meet the RF emission regulations, the use of directional antennas are envisaged. In the case of LEO, the satellite tracking is a difficult task due to the relative motion with the earth observer. Moreover the handheld tilt and pitch requires a fast tracking and an adaptive antenna array implemented on the device. Technology efforts in this direction have been made but the feeling is that there will be probably a separation between high and low bit-rate satellite terminals. Compact voice and low bit-rate terminals will operate on LEOs with omni directional antennas while laptop-sized data terminal will connect through planar adaptive arrays to GEO or MEO satellites.

\section{2) Access}

Dedicated circuit switched channels do not meet the digital satellite philosophy. The expensive transmission medium is shared and the access technique must provide flexibility and adaptability to traffic characteristics. Third generation communication systems are based on CDMA technology for multiple access [4]. The most innovative concept brought by CDMA is the "soft capacity"; the common medium can be loaded with users at the graceful expense of the overall transmission quality. Turbo coding and space-time diversity schemes [5] can be used to increase the channel utilization and protect the most valuable information.

For access from a limited number of satellite stations the TDMA-based access schemes still provide an efficient and reliable access: DVB-S access employs the TDMA scheme on GEO satellites.

\section{3) Transport}

The importance of a suitable transport has become essential for two main reasons: to support the Quality of Service and improve the efficiency.

The Internet protocol stack, TCP/IP, is now considered as a candidate for a global transport protocol. Though it is not designed to support QoS and real time traffic like voice and video, its extensions can efficiently support these features. With these consideration in mind the proposed transport formats for integrated (media and data traffic) satellite networks are basically three: ATM, IP and DVB. The last one is specific to broadcast services and can be only used at the data link layer of the protocol stack. IP and ATM have networking layers but the first one has been specifically designed for fast reliable terrestrial links. On satellite, errors during transmissions and long round-trip delays limit the performances. For this reason modified ATM cells are proposed for satellite links to optimize throughput and resiliency. 
IP, native or encapsulated over ATM or DVB, is considered the most interesting proposal. The main advantage is the natural integration with existing terrestrial networks, mainly IP based. The common IPv.4 protocol however misses some key features necessary to support next generation services. Quality of service, security, support for scalable multi-cast traffic, poor TCP performance over satellite are only a few problems that the scientific community is trying to solve. Several proposal and solutions are already on the table but the choice is often market driven, as IP versus ATM showed.

\section{4) All in Software!}

A special emphasis is due to a now consolidated implementation trend. The availability of faster DSP processors, large memories, fast analog-to-digital converters, lead us to imagine that most of the abovementioned technological functions could be shortly implemented in software. If this concept is well known at higher layers in the OSI stack, for physical stratum it represents a real revolution. Under the name of "Software Radio" fall the ability for a communication device to define all the functions in the transmission chain with software instructions. The impact of this technology is evident, a single device can implement several transmission methods, access schemes and transport protocols, simply by loading, or even downloading the appropriate code from the network. Recently announced ADCs able to sample at the notable speed of 1500 Msps open the door to this technology.

\section{TRENDS IN SERVICES}

In the recent years the explosive growth of Internet and, in parallel, the success of second-generation mobile cellular systems with an exponential number of users proposes a new interesting trend.

The interoperation between satellite systems, the existing and future mobile communication systems and the existing terrestrial infrastructures, Internet first of all but also ISDN and ATM, introduces the needs of new multimedia hybrid services defined as services that span different network technologies. High speed and high quality multimedia services are therefore expected with different quality of service (QoS) requirements.

The third generation mobile communication system Universal Mobile Telecommunications Service (UMTS) allows to overcome the bandwidth limitations and provides services with variable bit rate before and during the call, to satisfy the requirements of high speed customers.

The satellite component in the UMTS (S-UMTS) will provide the global coverage and roaming of UMTS users also in developing countries maintaining terminal compatibility and service flexibility.

The S-UMTS should be able to support user bit rate up to 144 $\mathrm{kb} / \mathrm{s}$. This bit rate is considered sufficient to offer multimedia application based on H.320, H.323, H.324 standards and also the MPEG-4 standard. Different BER and delivery delay are provided according the required service: for speech it is required BER equal to $10^{-3}$ and $400 \mathrm{~ms}$ maximum delay, for data application BER of $10^{-9}$ and $400 \mathrm{~ms}$ maximum delay for videoconference but also few seconds for Internet access and few minutes for e-mail delivery.

A satellite network is inherently a broadcast system and up to now the global market for digital-to-home (DTH) satellite is the delivery of broadcast television. Broadcasting and data application such as pay-per-view, home shopping, electronic commerce and on line delivery data services require a lowdata-rate return path, typically a phone line.

New multimedia applications including high-speed Internet, IP multicast, distance education and telemedicine require higher and flexible data rate an two-way communications.

The interactive channel for multimedia satellite networks (ICMSN) is under standardization by ETSI with a forward link based on a digital video broadcast (DVB) MPEG 2 data format up to $45 \mathrm{Mb} / \mathrm{s}$ and a return link using a multifrequency -time division multiple access (MF-TDMA) scheme providing between 144kbps and $2.048 \mathrm{Mbps}$.

Commissioned by the European Commission EC-DGXIII within the frame of the ACTS 4th Framework Program, the ISIS (Interactive Satellite multimedia Information System) project and the Galenos (Generic Advanced Low cost transEuropean Network Over Satellite) project have already utilized and tested a return channel suitable to provide interactive multimedia services.

The ISIS system simultaneously uses a dual band terminal, $\mathrm{Ku}$ frequency band for a high rate forward link and $\mathrm{Ka}$ frequency band for the return link to provide a transmitter/receiver terminal at low- cost, with small size, easily transportable and installed.

The ISIS system simultaneously uses a dual band terminal, $\mathrm{Ku}$ frequency band for a high rate forward link and $\mathrm{Ka}$ frequency band for the return link to provide a transmitter/receiver terminal at low- cost, with small size, easily transportable and installed and to offer interactive services at the residential end-users typically developed in INTERNET (ftp, email, browsing services, Distribution of Electronic Newspapers, Team-working, Tele-education, Telemedicine) on the satellite DVB environment.

The Galenos network uses a multicast connection (forward and return links in $\mathrm{Ku}$ band) by Eutelsat to promote Telemedicine applications among many Hospitals (Berlin, Toulouse, Marseilles, Naples, Florence Matera, Athens, Samos, Sophia, Tunis) in the Mediterranean area

\section{1) Tele-education Application}

Communications for remote education, a typical asymmetrical application, can be categorized as follows:

1) Broadcast type communications, by which the students can received the lectures in wide areas also directly to own home;

2) Interactive communications: based on videoconference it allows two-way communication 
from students and teacher and viceversa. The students and the teacher can interact remotely such as they was in the same class-room. The teacher can control the students, can formulate questions or propose tests that they must resolve on line.

3) Self-learning, in which the students can retrieve learning materials remotely.

In Isis project an experiment of self-learning based on Web distribution of hipertexts with interactive live video is performed providing good results in terms of QoS.

\section{2) Tele-Medicine Application}

Tele-Medicine aims to provide remote medical care services to distant communities enhancing the autonomy of hospitals dislocated in remote sites and family physicians, increasing efficiency and allowing quality assessment.

A telecommunication network can make the patient data available in remote sites, enhancing the co-operation between physicians of different institutions.

The main objectives of this application are:

- Medical Database Access: it permits the access a common data base of multimedia data (exams, anamnesis, therapy, radiography) relevant to any patient in any institution connected to the network. The medical protocols and guidelines for decease treatment and surgery can be shared, to a medical diagnostic images database

- Remote Expert Consultation: it provides interactive image transmission showing various medical diagnostic modalities with co-operative tools, among physicians and specialists that exchange information and opinion about patient cases to obtain diagnosis in real time and for planning the treatment to follow.

The tele-medicine applications require an high resolution of medical images that the large bandwidth of satellite systems can guarantee. The Medical Data Base Access requires a typical point-to multipoint communication and it can have advantages by the satellite broadcast distribution.

The Remote Consulting requires on line communication at high speed especially in case of emergencies or surgery consultation, therefore a IMCS channel is necessary.

On ISIS satellite network a Medical Environment for Diagnostic Images (MEDI) has been developed and tested MEDI is a WEB application based on HTML pages, JAVA client front end (applets), JAVA servers and on the TCP/IP protocol and it is particularly suitable to broadcast connections for its clients/server architecture.

The physicians can communicate remotely and open medical sessions exchanging radiographic images in the Papyrus 3.0 format (in agreement with DICOM 3.0 part 10 standard), sharing tools for co-operative diagnosis or to planning the treatment to follow.
The GALENOS promoted the exchange and test of different tele-medicine applications among the participant hospitals. In particular, the Careggi Hospital- Florence and the Ropbert-Roessle-Hospital at MDC, Charité, Berlin opened different sessions of teleconsult according the connection scheme shown in Fig.2 These hospitals used the WinVicos (Wavelet based interactive Video communication system) application by the OP 2000 at RRK/MDC, Charité, Berlin. The WinVicos was a high-end, interactive video conference system providing real-time video, still-images and audio transmission designed for teleconsultation, second opinion, etc. WinVicos employs a hybrid speed-optimised waveletcodec for video compression and therefore guarantees a high resolution of the images. Audio compression is done by the MPEG Layer 3 compression algorithm

The video conference partners can see each other, talk, exchange images and even use remote-pointers to point at certain details.

Additional diagnosis tools are tested in different demonstrations such as:

- Virtual reality applications as $3 \mathrm{D}$ reconstruction for patient CT data for planning of the surgical treatment

- Demonstration of the surgical simulator in Berlin (Cyberspace surgery)

- Transmission of high resolution pictures (still or moved images) and co-work with the virtual pointer

- Full control of a microscope for histopathological investigations (microscope location in Berlin; control via the terrestrial $128 \mathrm{Kbps}$ Euro-ISDN Network from Florence)

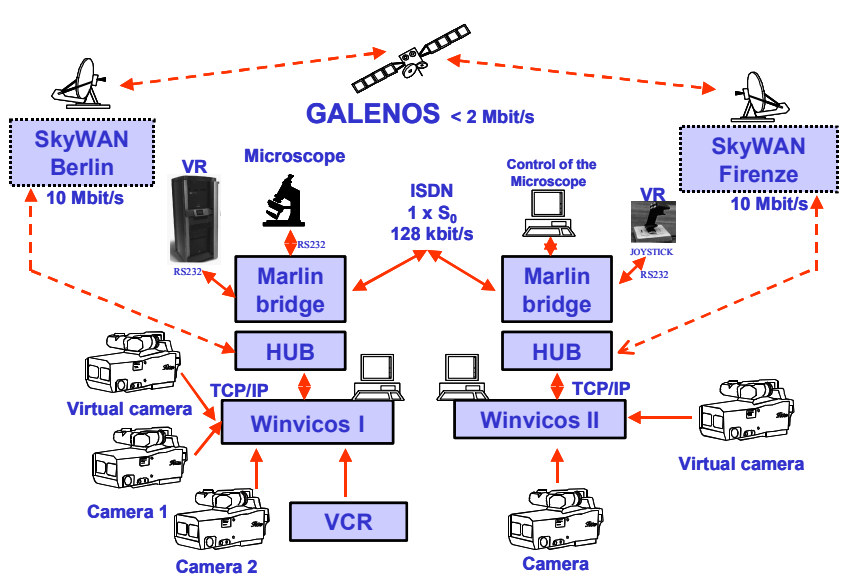

Fig.2 Eutelsat connection for tele-medicine applications in the Galenos project. 


\section{TRENDS IN BUSINESS OPPORTUNITIES}

The large coverage and the ability to reach a large number of users at the same time provide also novel economical opportunities for companies.

The Business-to-Customer telecommunication market is now spilt into two major lines: the provision of services by a fixed terrestrial network and the mobile communication system. Though they are interoperating at a technical level, they differ substantially for their economical aspect. Terrestrial networks constitutes an investment on sites, rather than on people. A wideband fiber deployment in a small geographical area forces the operator to tie to a particular set of users, since continuous activations and interruptions of services are costly and risky operations. Mobile operators, on the other hand, try to serve as many people as possible by limiting the service offer in terms of bandwidth and quality. Satellite system can provide almost all the service flavors of a terrestrial deployment with the flexibility of a mobile network. Another key issue is the enormous increase of the contents in the Internet. Companies advertisements, catalogues, on-line showrooms and electronic commerce portals tend to saturate the present Internet capacity. In this case a highly asymmetric data distribution network, i.e. IP on DVB-S network, provides all the necessary capacity to a large number of residential users with a high efficiency due to the quasi broadcast nature of the service.

The Business-to-Business market well be also radically affected by the use of satellite networks. Large companies often have the need to connect occasionally different sites in different countries. Internet, however, does not provide a satisfactory level of quality for data exchange and real time services. ISDN and other circuit switched solutions are extremely expensive if the required bandwidth is large. Satellites can provide a relatively large bandwidth sold on a per-consumption basis, in every potential site within the coverage area.

\section{OPEN RESEARCH TOPICS}

Several open issues still require a considerable effort from the scientific community. The main problems affecting a satellite systems have been addressed in the past. Evolutionary, rather than revolutionary contributions are envisaged, especially to obtain an high level of integration between various networks with different transport characteristics.

At the physical level substantial improvements in the efficiency of the radio link between the earth terminals and the satellite are attended. The use of 2D planar antenna arrays, in conjunction with complex signal processing algorithm can reduce the earth terminal and optimize the transmission power. Also on satellites, after launch antenna deployment can provide GEO systems with higher gains and smaller and more precise spot-beams on earth surface.
New frequencies, like $\mathrm{W}$ band $(90 \mathrm{GHz})$, are explored for communications.

At the transport level are currently studied improvements on protocols for the characteristics of satellite communication environments. Transports protocols fully integrated with the TCP/IP protocol stack of the Internet are explored. Security is another important element. The signal from satellites are accessible by anyone in the coverage area, so security mechanisms must be adopted to prevent undesired listening or hacking of functionalities.

At application and service levels several EC activities have been founded to promote the use of satellites in the Euro area. The main objective is to share in a short time cultural and social resources among the countries, in order to promote the countries without a consolidated industrial history.

\section{ACKNOWLEDGEMENT}

The authors would like to thank the COST252 participants for the co-operation and the useful contribution on satellite system researches.

\section{REFERENCES}

E. Del Re, "A coordinated European effort for the definition of a satellite integrated environment for future mobile communications", IEEE Communications Magazine, February 1996

[2] http://www.tbs-satellite.com/tse/online/prog iridium.html

[3] David Project, http://david.eln.uniroma2.it/, RSE Experiment.

[4] 3GPP - Third Generation Partnership Project, http://www.3gpp.org/

[5] Hochwald, B.; Marzetta, T.L.; Papadias, C.B., "A transmitter diversity scheme for wideband CDMA systems based on space-time spreading", IEEE Journal on Selected Areas in Communications, Volume: 19 Issue: 1 , Jan 2001, Page(s): $48-60$

[6] P.Taaghol, B.Evans, E.Buracchini, R.De Gaudenzi, G.Gallinaro ,J.HoLee, C.Gu Kang, "Satellite UMTS/IMT2000 W CDMA Air interfaces” IEEE Comm.Magazine,September 1999

[7] E.Del Re, L.Pierucci, "Next-generation Mobile satellite Networks" Accepted/to appear on IEEE Communications Magazine

[8] E.Del Re "Evolution of mobile satellite communications systems" in press Springer Verlag November 2001 